\title{
The Role of Non-Verbal Interaction in a Short-Term Psychotherapy: Preliminary Analysis and Assessment of Paralinguistic Aspects
}

\author{
Diego Rocco ${ }^{\natural}$, Rachele Mariani ${ }^{2}, \&$ Diego Zanelli ${ }^{1}$
}

\begin{abstract}
Analysis at a paralinguistic level of communication, already conceptualized within the multiple code theory, would appear to be very important in order to fully describe the quality of the patient-therapist relationship. In this study the therapeutic process and microprocess are analyzed taking into consideration a specific paraverbal aspect (speech rate) present in patient and therapist's communication. More specifically, in this paper we aim to investigate the relationship between the speech rate of both patient and therapist with the linguistic aspects of their referential process as obtained by the IDAAP dictionaries, relating to three sessions belonging to different phases of the psychotherapy. The results show that there are many significant correlations between the considered values. These findings are interpreted as an expression of the alignment between patient and therapist which can be linked to the outcome of the psychotherapy.
\end{abstract}

Keywords: paralinguistic aspects, relationship, process, short-term psychotherapy

Over the last few decades the field of psychotherapy has seen a gradual convergence of technical and relational aspects of the therapeutic setting: These two aspects have gradually become inextricably linked, each one depending on the existence of the other (Karasu, 1986).

This conceptualization of the psychotherapy process can be taken much further, to the point that "the interpersonal and spontaneous processes between patient and therapist deserve more attention than as merely a necessary precondition for the effectiveness of technical interventions and should be considered the central factor of change themselves" (Merten, 2005, p. 325).

The relational component seems to be "highly relevant in establishing and shaping the therapeutic rela-

\footnotetext{
${ }^{1}$ Department of Philosophy, Sociology, Education and Applied Psychology, University of Padua.

${ }^{2}$ Department of Dynamic and Clinical Psychology, Sapienza University of Rome.

$\triangle$ Correspondence concerning this article should be addressed to Diego Rocco, University of Padua, c/o LIRIPAC, Via Belzoni, 80, 35100 Padova. E-mail: diego.rocco@unipd.it
}

tionship" and therefore "one of the main goals in psychotherapy research is to investigate and determine those processes that cause change within the therapeutic relationship" (Benecke, Peham, \& BänningerHuber, 2005, p. 81).

From this point of view, the processes that could be related to the change in a therapeutic relationship are self-regulating processes and interactive regulatory processes, which are present in the relational approach in psychoanalysis (e.g., Mitchell, 2000; Stolorow \& Atwood, 1992) and in Infant Research (Beebe \& Lachmann, 2002; Tronick, 1998). Interactive regulation "means that contingencies flow in both directions between partners. That is, the behavior of each partner is contingent on, 'influenced' by, or predicted by, that of the other. [....] The person experiences both influencing and being influenced by the partner" (Beebe \& Lachmann, 2002, p. 27) while the term self-regulating processes is used "to denote the capacity of the partners to regulate their respective states. From birth onward, selfregulation refers to the management of arousal, the maintenance of alertness, the ability to dampen arousal in the face of overstimulation, and the capacity to inhibit behavioral expression" (p. 28). 
It is through these complex dynamics that the therapeutic process is realized and the therapeutic action is developed.

The relational aspect can be linked, more than to the contents of communication, to its implicit/nonverbal aspects (paralinguistic aspects), which seem to be involved in determining the perceived relational climate by both the patient and the therapist (Stern, 2004). Beebe and Lachmann (2002) claim that the "basic processes of interaction at non-verbal level remain so similar across the life span" (p. 23) that their continued influence on a relationship represents "an essential dimension of therapeutic action" (p. 57). They say, for instance, that "if the analyst can 'read' the nonverbal communications, from the ongoing stream of behavior in both the patient and herself, they have the potential to alert her, often well before the verbal process can do so, to subtle difficulties in the engagement" (p. 129).

The same authors argue that "timing coordination is an early communication system in infancy and a scaffolding for the subsequent development of social communication" (Beebe \& Lachmann, 2002, p. 106), and it is on this basis that one can stress the fact that in adults the processes of self-regulation and interactive regulation occur simultaneously, both at the level of implicit and explicit communication, thus resulting in much more complex, and at the same time clinically richer, communication (for a schematic overview of these concepts see Beebe \& Lachmann, 2002, p. 35). Drawing on these considerations, Tomicic, Martinez, Altimir, Bauer, and Reinoso (2009) underline the importance of assessing the non-verbal coordination in patient/therapist dyad, and to achieve this goal they recommend the use of specific software. The authors say that the results obtained should allow the verification of Beebe and Lachmann's insight concerning the presence, in adult treatment, of the same timing coordination found in mother/child dyads.

Despite all of this, the analysis of voice quality for communicative processes or, in other words, the effects of emotion on speech intonation and tempo, has received little attention at the moment. Moneta, Penna, Loyola, Buchheim, and Kächele (2008), in a pilot study examined the voice quality as spectral parameters extracted from vowels during a single semistructured interview. They found, as predicted, that the pronunciation of the vowels in the word "mutter" (mother, in German) has different vocal features related to the subject's attachment style (secure vs preoccupied). The authors interpreted this data as an example of the influence that affect and emotions have on the paralinguistic features of verbal production.

Horowitz, Sampson, Siegelman, Wolfson, and Weiss (1975) studied, in a psychoanalytic treatment, the patient's discomfort by analyzing his speech disruption while pronouncing warded off contents. They found a significant relationship between this paralinguistic parameter and the kind of content (warded off vs. not warded off).
Some studies (Bauer, Tomicic, Martinez, Reinoso, \& Guzman, 2008) have focused on the relevance of the features of the therapist's voice, others (Rice $\&$ Kerr, 1986; Rice \& Wagstaff, 1967; Wiesman \& Rice, 1989) have studied the quality of patient and therapist's voices by examining it in relationship to psychotherapy process and outcome.

Our point of view is that in order to venture into a successful empirical analysis of the relational aspects of the therapeutic process, it is necessary to use a theoretical model which provides and includes within it the possibility of analyzing contemporaneously the explicit and implicit communication channels, considering them as integrated and synergistic.

The work carried out by Wilma Bucci articulates this in in-depth theoretical and clinical terms.

\section{The referential activity and implicit aspects of communication}

Bucci has developed an analytical model of the psychotherapeutic process in psychoanalysis by integrating the constructs of psychoanalysis (in particular the construct of the primary process and secondary process) with those derived from studies from cognitive psychology. Thus Bucci defined the multiple code theory (1985, 1997a, 1997b, 1999), in which the author identifies three ways of processing information, subsymbolic, symbolic non-verbal and symbolic verbal (for the characteristics of these three systems of information processing we refer to the work, in this Special Issue, of Mariani and De Coro, 2013). The process which allows the integration of these three formats of information processing is referred to by Bucci as the referential process, a complex cognitive function which, during the course of psychotherapy, must be activated in order to enable the patient to reconstruct those connections between experiences and words which were previously dissociated.

The analysis and the assessment of this process during a psychotherapy is permitted by the referential activity (RA) methodology, created by Bucci and Kabakalian-McKey (2004). This methodology foresees a breakdown of the session transcription into idea units (IU, or distinct parts of the session, each of which is characterized by a focus), subsequently the awarding of points on four scales, by more than one judge, for each IU. The four scales are: concreteness, based on "degree of perceptual or sensory quality, including references to all sense modalities, action, and bodily experience," specificity, the amount of detail such as "explicit descriptions of persons, objects, places, or events," clarity, the extent to which an image is "seen through the language; how well-focused the linguistic image is judged to be" and imagery, "the degree to which the language evokes corresponding experience in the reader or hearer" (Bucci, 1997a, pp. 188-189). Bucci has identified these four scales in her studies as being most appropriate to assess the extent to which emotions and images are expressed in words. In this 
way, the data obtained also enables the detection of CONIM (which, equating to the average of the concreteness and imagery scales, can be used as the production level of sensory mental representation in the patient's language) and CLASP (which, being the average of the clarity and specificity scales, can provide an indicator of the formal organization of the patient's speech), as well as the total score of referential activity (RA, equal to the average of the four scales).

In addition to the three channels of information processing mentioned above (subsymbolic, symbolic non-verbal and symbolic verbal), Bucci points out that in the process of subsymbolic activation there also exist certain elements of a subsymbolic verbal code (Bucci, 2011). The latter refers to paralinguistic aspects such as voice tone, intensity, and silences. Bucci claims that "paralinguistic features of language may be but are not necessarily connected to symbolic language, and may also carry communicative information in their own channels. [....] The role of such subsymbolic verbal (or vocal) processing, which might constitute a fourth processing format, is not considered as such in this formulation of the multiple code theory and needs to be addressed further in subsequent work" (Bucci, 1997a, p. 176). This format is very important as it is "particularly dominant in emotional communication. [....] Dissonance in communication of emotional meanings occurs when the information carried in the linguistic and paralinguistic tracks do not correspond" (p. 176).

In the clinical setting, this fourth format can be of fundamental importance to the patient-therapist dynamics. It is also through the unconscious perception of variations in the patient's subsymbolic verbal channels that the therapist (while paying attention to the explicit meanings conveyed by the semantic channel) can modulate his subsymbolic channel, and with a good understanding of the internal state of the patient, can encourage the broadening of his consciousness.

With the aim of integrating, on the one hand Beebe and Lachmann's (2002) ideas concerning the role of non-verbal interactions and timing coordination on clinical exchange, and on the other hand Bucci's affirmation concerning the existence of a subsymbolic verbal code, Rocco $(2005,2008)$ has conducted research concerning a paralinguistic dimension, the speech rate (SR), which, in the context of the model proposed by Bucci, could be considered to be a parameter that belongs to what Bucci defines as subsymbolic verbal codes.

Among the possible non-verbal parameters, the SR was chosen for two reasons: its detection and subsequent analysis do not require much adaptation of the setting, since only a small digital recorder is needed (although this could also be true for other parameters, i.e. pitch) but, more importantly, this parameter is able to detect variations of the SR parameter that are consistent with Bucci's theoretical model, since its underlying assumption is that certain features of speech production analyzed following Bucci's method would require more processing time. An example is when referential connections between language and the underlying emotional core are activated (this should happen in the activation of the subsymbolic phase which is then followed by the referential connection phase). A longer period of time taken would necessarily depend upon the process of symbolization (Bucci, 1997a).

These studies have investigated, in patient verbal production, how the SR varies according to the features of referential activity detected by manual methodology (comparing moments in which the patient was using more evocative language-high CONIM scores-to others in which the language was more organized and less emotive). Also studied was the presence of coordination between patient and therapist's SR in different psychotherapy sessions (session with good alliance vs bad alliance; sessions with good access to emotions vs sessions with limited access to emotions). By means of correlations, the following results were obtained:

(i) a significant negative correlation between patient's SR and the CONIM score, while the correlations were positive between CLASP and SR (Rocco, 2005). These results were in line with Bucci's theory;

(ii) a significant correlation occurred between the therapist's SR and both the scores of the patient's RA, the CONIM and CLASP scores, and also the scores of the four scales (Rocco, 2005, 2008);

(iii) finally, in different sessions with different patients, a correlation emerged between patient's SR and that of the therapist (the features of the correlations depended on the RA trends of the sessions considered; Rocco, 2008).

Despite the results obtained in these two studies, we think that there are other aspects that should be examined. For instance the correlation between the patient's SR and the therapist's RA, which could confirm an interactive regulation, was not studied.

A further aspect to consider is that the RA assessement now has a computer methodology (the IDAAP system; Italian Discourse Attributes Analysis Program; De Coro, Mariani, et al., 2004; De Coro, Ortu, et al., 2004) that provides us with new information that the manual RA version does not, thus allowing us to conduct more in-depth theoretical and clinical analyses.

Indeed, while the manual procedure provides only four scales and the two subscales CONIM and CLASP, with the four IDAAP dictionaries it is possible to access data from ten different scales; it is also possible to extract from the dictionaries a broad array of data concerning the covariation between the different scales, which allows the production of 38 quantitative variables.

A further clear advantage of computer scoring is that 
using the dictionaries it is also possible to calculate the same 38 variables for the therapist's verbal production; using manual scoring this is not always possible.

It is also for these reasons, and to confirm the data from the works cited, that in this study we assess the correlations between SR and the evaluation of RA obtained through the IDAAP system in a sample of psychotherapy sessions.

The aims of this study are:

(1) To verify the correlation between the patient's SR and the scores obtained by applying the four dictionaries to his verbal production. The expectation is that the correlation will be negative with scales that detect subsymbolic aspects, while the correlation will be positive with dictionaries concerning words expressing formal aspects of the language. We expect these results because the verbalization of subsymbolic aspects should require more time for processing compared to the processing of formal aspects alone. We also expect the presence of a correlation between SR and the covariation between different RA scales. More specifically, the correlation sign (positive or negative), will depend on which scales covary (for instance if the covariation is positive, expressing integration between emotional and formal aspects, a negative correlation with $S R$ is expected, since a longer processing time would be needed).

(2) To verify the presence of correlation between the therapist's SR and data from the application of the four dictionaries to the patient's verbal production. This correlation could be due to the dynamics of interactive regulation which, from this point of view, could involve the therapist's referential process, which, in turn, is influenced by the characteristic of the patient's referential process. We expect that if the patient is using dictionaries activating his referential process, the therapist's SR will be lower. Moreover we expect some correlation between the SR and the dictionaries' covariation; the correlation sign will depend on which scales are subject to covariation and the meaning of the covariation in terms of RA following the same consideration above.

(3) To verify the presence of correlation between the patient's SR and data from the application of the dictionaries to the therapist's verbal production. This hypothesis is closely connected to the previous one. Since we want to verify the presence of an interactive regulation between patient and therapist, we expect that there will be a significant correlation between the SR of the therapist and that of the patient, each as an expression of their own referential process, and the verbal expression of the other. We therefore expect there to be a circular reciprocal influence expressed by the presence of significant correlations. As for the previous hypothesis, we expect that there could be a referential process modulation present in the patient which would be dependent upon the quality of verbal production, and therefore the referential process, of the therapist (as for the previous hypothesis, the correlation might mean that it is the therapist who modulates his verbal production in a way that the dictionaries can detect). As previously underlined the direction of the correlation could vary in relation to the specific dictionary utilized or in relation to the covariation considered.

(4) To verify the presence of positive correlations between patient and therapist's SRs, when considering all sessions together and some of the individual sessions. These expectations are linked to suggestions regarding the coordinates of temporal timing arising from infant research, where studies of the mother-child dyad have focused on different kinds of coordination of identifying interpersonal timing, assuming that an optimal coordination involves a moderate level of bidirectional coordination (the coordination between the timing of each verbal production is present at times and not at others, Jaffe, Beebe, Feldstein, Crown, \& Jasnow, 2001). When a continuously high coordination is present, the child must make a considerable effort to anticipate the mother's timing. In the same way we expect that the relation between SR aspects of patient and therapist should have the same characteristic, that is using the full range of possible timing coordinations, thereby avoiding an excessive uni-directional tuning, from patient to therapist.

In this research we do not analyze the relationship between the therapist's SR and the scores obtained by applying the four dictionaries to therapist's verbal production, because we mainly concentrate on the features of the patient's referential process and on the way it is related to the therapist's verbal production.

Even though there are ten scores taken from the four dictionaries of the IDAAP system, plus 28 quantitative scores derived from the covariation of the scales, we do not expect there to be significant correlation between SR and all these scores. Rather, we expect that, more often than not, both the single scales and the covariation scores that correlate significantly with SR will concern scores of referential activity or scores that express the formal organization of the discourse.

\section{Method}

\section{Data}

The methodology described was applied to three sessions of a short-term psychotherapy: the first session, the middle session (number seven) and the final session. The central, seventh session lasts one and a half hours, and this particular duration is in line with the shortterm psychotherapy model, and related technique, pro- 
posed by Davanloo (1990).

The patient, C, was a 21-year-old male university student, of medium socio-economic level, who reported a sexual problem he had had for three years and some difficulties in relationships as well. He received 14 sessions of psychodynamic short-term psychotherapy with an approach based on the work of Davanloo (1990), integrated with suggestions from Fosha (2000).

Mr. C's treatment is considered to be successful, according to changes in symptom profile measured by the Symptom Check List (SCL-90-R; Derogatis, 1983), and personality profile, assessed by the ShedlerWesten Assessment Procedure (SWAP-200; Westen, Shedler, \& Lingiardi, 2003).

The psychotherapy sessions were held by a male psychotherapist with over ten years of experience. All the sessions were audio recorded with the patient's informed consent.

\section{Procedures}

The transcripts of the patient's three sessions were segmented into Idea Units (IUs, distinct parts of the session, each of which is characterized by shift in focus of a narrative or any discourse) following Bucci and Kabasakalian-McKay's (2004) instructions for segmenting a narrative. Two expert judges separately identified the IU boundaries. Initially there was $82 \%$ agreement on the IUs for which they found the same beginning and the same ending. For the IUs for which they had no agreement, there was a discussion phase, as described in the literature (Bucci \& KabasakalianMcKay, 2004; Fogliato, Strappa, Branchini, \& Rapisarda, 2009), in which they explained to each other the reason for the choice taken, until agreement was reached. This procedure resulted in session number 1 having 19 IUs, session number 7 having 49 IUs and the final session having 21 IUs.

Subdividing the transcripts into IUs allows for the minimization of RA fluctuation within the unit, because IUs are designed to capture a single "shot," carrying a distinct idea. Each shift in meaning or focus of the narrative is marked as the beginning of a new IU.

The sessions were audio recorded with a digital recorder placed at the same distance from the patient and the therapist, in order to have a good voice quality from both. Subsequently the sessions were transcribed verbatim following the Italian rules for transcription (Mergenthaler, Freni, Giampieri, \& Ferrari 1998) and later adapted to the rules needed for elaboration by the IDAAP system (see next paragraph).

\section{Measurement}

The IDAAP system and computerized dictionaries. The IDAAP system was designed to read texts, compare them word by word with its dictionaries and calculate a weighted average of the dictionary scores attributed to each speaker, for each turn of speech, for each text and for each session. This computing allows us to obtain averages for a micro and macro analysis of sessions, and also allows the assessment of the extent of the referential process, by applying its four dictionaries. Each dictionary produces scores for one or more scales, ten in total.

\section{Italian-Weighted Referential Activity Dictionary (I- WRAD):}

(1) Mean Italian Weighted Referential Activity Dictionary (MIWRAD2): Mean of referential activity.

(2) Mean High Italian Weighted Referential Activity Dictionary (MHIWRAD2) scale): Measure of the intensity: moment until the speaker is using a high RA.

(3) Mean High Italian Weighted Referential Activity Dictionary (HPIWRAD2): Proportion of speech in which the speaker is in a high AR mode.

(4) Mean Italian Sens Somatic (MISenS): Mean of somatic words.

\section{Italian-Affects Dictionary:}

(5) Mean Italian Affect Positive (MIAffP): Mean of Positive Affects.

(6) Mean Italian Affect Negative (MIAffN): Mean of Negative Affects.

(7) Mean Italian Affect Neutral (MIAffZ): Mean of Neutral Affects.

(8) Mean Italian Affect Sum (MIAffS): Mean of the sum of all the Affects.

\section{Italian-Reflection Dictionary:}

(9) Mean Italian Reflection (MIRef): Mean of Reflection words.

\section{Italian-Disfluency Dictionary:}

(10) Mean Italian Disfuency (MIDF): Mean of Disfluency.

Each of these scales can have a score between 0 and 1 . For the scale MIWRAD2 (mean of RA), .5 represents a neutral score (this is significant because if the score is over .5 it is an indication of the extent to which the speaker is engaged in referential activity; if the score is under .5 is an indication of the extent to which the speaker is doing something else).

The data of the covariations are calculated from each pair of all the scales in the above list (excluding MHIWRAD2 and HPIWRAD2). The covariation between two dictionary scores is comparable to the correlation coefficient, but does not have the same statistical meaning. Covariation is a measure of the degree to which words belonging to any given pair of dictionaries are simultaneously considered to move together or in different directions, and provide information that is important in understanding the therapeutic process (see, in this issue, Mariani \& De Coro, 2013). 
The covariation index between paired scales varies between -1 and +1 .

In order to elaborate the text of the sessions with the four dictionaries of the IDAAP system it was necessary to prepare the transcripts of the sessions in line with the following rules:

(1) the definition of IUs;

(2) identification of the speaker (patient or therapist);

(3) the spelling of unusual, made up, or incomplete words, etc.

The sessions were then processed using the IDAAP system which gave rise to an output for each IU of each session, producing an average score for each of the ten scales deriving from four dictionaries, as well as the covariance scores which take the individual scales into account, combined in pairs.

The unit of analysis for the application of the IDAAP dictionaries was the IU. The IDAAP system provided, by the analysis of the words belonging to each IU, a mean for each scale (e.g., mean of reflection words, mean of disfluency, etc.). The term "weighted," referring to the dictionaries, means that while most text analysis programs match words in a sample to a dictionary, count the number of occurrences and divide by the total word count to calculate a proportion, the IDAAP also has the capacity to calculate a score based on a weight assigned to each word in a dictionary.

The speech rate calculation. The use of PRAAT (Boersma \& Weenink, 2012), a specific computer program that enables visualizing, annotating and analyzing of sound objects in terms of their acoustic properties (e.g., frequency, pitch, time) for the analysis of speech and the transcription of the session, allowed us to calculate the SR (Rocco, 2005). For each IU, the patient's SR was calculated as the number of syllables per second. The syllables were identified and counted by a judge following the rules of Italian grammar.

For IUs in which only the patient talked, the calculation of SR involved measuring the time necessary for the patient to produce the phrases uttered in each individual IU. Then, the syllables within the considered phrases were counted, and finally the ratio between the number of syllables pronounced and the time taken was calculated. This procedure allowed the identification of the mean number of syllables per second for each IU.

A turn of speech is defined as the uninterrupted verbal production of one participant which ends when the other participant starts to speak. For IUs in which both patient and therapist spoke (with one or more turns), SR was calculated in the following way. For patients, the total number of syllables within each phrase and the time taken to utter each phrase were calculated. Each phrase was considered to start with the first word uttered and to finish with the last word uttered. Any pauses between turns (i.e., between patient and therapist) were not counted. For each IU, the number of syllables and the time taken to utter the phrases was calculated (summing all the uttered syllables and all the time taken). The two figures obtained were then used to calculate the patient's SR for that particular IU. The same procedure was repeated in order to obtain the therapist SR. In the few moments (no more than four in each session) in which patient and therapist were overlapping (i.e., talking simultaneously), if the voices were sufficiently clear to permit the technician to understand the moment in which the patient (or the therapist, or both) stopped speaking, then the methodology was applied as described. If the voices were not clear, the technician calculated the SR of the patient (or therapist, or both) taking into consideration only the words identified. Pauses of 3 seconds or less in the phrases pronounced were included in our measurement of the total time taken to produce the phrases. Pauses which exceeded 3 seconds were considered as three-second-pauses. In other words, if there was a pause of, for instance, 3.5 seconds, this was recorded as a three-second-pause. It was necessary to establish this cut-off point in order to prevent the SR scores being compromised by excessively long pauses that were not necessarily due to cognitive or emotional processing, but instead, for example, to crying. The pauses between patient and therapist's turns were not counted.

The SR was calculated to two decimal points for each IU both for the therapist and the patient's verbal production. For each IU, the number of syllables was calculated, and the SR was computed as the number of syllables divided by the duration in seconds, as previously described.

After having applied the measures described above (concerning the IDAAP measures and the SR calculation) we obtained the following data for each IU of each session:

(i) the scores of the 10 IDAAP scales both for the patient and for the therapist;

(ii) the covariance scores obtained by the pair of individual scales;

(iii) the SR scores both for the patient and the therapist.

Considering the limited number of sessions analyzed, this is to be considered a preliminary study.

\section{Results}

The data relating to the four working hypotheses is presented below. A Spearman rank correlation was applied to the three sessions, between the data coming from the IDAAP scales applied to verbal expression both of the patient and the therapist and the SR scores of both patient and therapist. Spearman's correlation was chosen because parametric assumptions of normality were not met for the samples, moreover the sample (number of IUs) is quite small. For each hypothesis only significant correlations have been reported. 
Table 1. Values of Spearman's correlation between patient's SR and patient's IDAAP scores in the initial session (19 IUs), central session (49 IUs) and final session (21 IUs)

\begin{tabular}{|c|c|c|c|c|c|c|c|}
\hline & \multicolumn{2}{|c|}{ Initial session } & \multicolumn{3}{|c|}{ Central session } & \multicolumn{2}{|c|}{ Final session } \\
\hline & MISens & MIWRAD2 & MIRef & IAffN/ISens & IAffP/IRef & IAffN/IWRAD2 & IAffS/IDF \\
\hline SR & $-.396^{*}$ & $-.465^{*}$ & $.238^{*}$ & $.243^{*}$ & .283 & $-.376^{*}$ & $-.504^{* *}$ \\
\hline
\end{tabular}

Note. MISens = Mean of somatic words. MIWRAD2 = Mean of referential activity. MIRef $=$ Mean of reflection words. IAffN = Mean of negative affects. IAffP $=$ Mean of positive affects. IAffS = Mean of sum of affects. IDF $=$ Mean of disfluency. $S R=$ Speech rate.

${ }^{*} p<.05 ;{ }^{* *} p<.01$

Hypothesis 1: Relation between the patient's SR and RA. With the first working hypothesis we wanted to verify the presence of a correlation between the patient's SR and the scores of the four IDAAP dictionaries relating to his verbal expression. The following table (Table 1) shows only the values of the significant correlations for each of the three sessions.

In Table 1 we can see that, for the initial session, there are two significant negative correlations. From these it is clear that the patient's speech rate decreases when he increases both the amount of referential activity (MIWRAD2) and the use of words relating to emotional, physical or sensory experience (MISenS).

For the central session there is a significant positive correlation with the words belonging to the dictionary of reflective words (reflection dictionary), that is, the dictionary containing words that indicate, or refer to, the act of thinking. Two other correlations appear, this time with the co-variance of two scales. The first is a negative correlation with the covariation between the dictionary of negative affect and that of sensosomatic words. This indicates that when the words belonging to these two dictionaries, (which seem to express emotional and visceral aspects of significance to the background and problems presented by the patient), show positive co-variance, his SR decreases, subsequently increasing significantly if the covariation becomes negative. The second, on the other hand, is a positive correlation between SR and the covariation between terms contained in the dictionary of positive affects and words from the reflection dictionary. It therefore seems that when the covariation between these two dictionaries increases, meaning when the patient talks about positive affects using words belonging to the reflection dictionary, he speaks faster, whereas when these two scales diverge, his speech slows down (negative covariation).

Finally, for the session belonging to the final phase of treatment, there are two negative correlations with two covariations. In particular, these figures suggest that the patient speaks more slowly when his speech output simultaneously increases in both the scale of negative affect and that of referential activity, and when the scale of overall affect covaries positively with that of disfluency.

Hypothesis 2: Relation between the therapist's SR and the patient's RA. With the second working hypothesis we wanted to verify the presence of significant correlations between the therapist's SR and the data derived from the application of the four IDAAP computerized dictionaries on the patient's verbal expression. See below (Tables 2 and 3 ) the values of significant correlations in the three sessions.

It can be seen how in the initial session the therapist's SR correlates positively with the covariation of negative affects and reflexive words (this means that the therapist's SR is faster when the patient expresses

Table 2. Values of Spearman's correlation between therapist's SR and patient's IDAAP scores in initial session (19 IUs) and central session (49 IUs)

\begin{tabular}{|c|c|c|c|c|c|c|c|}
\hline & \multicolumn{2}{|c|}{ Initial session } & \multicolumn{5}{|c|}{ Central session } \\
\hline & IAffN/IRef & IAffP/IWRAD2 & MISens & MIWRAD2 & IAffP/IAffS & IAffP/IAffZ & IAffS/IDF \\
\hline SR & $.495^{*}$ & $-.479^{*}$ & $-.352^{* *}$ & $-.352^{*}$ & $.283^{*}$ & $.388^{* *}$ & $-.328^{*}$ \\
\hline
\end{tabular}


Table 3. Values of Spearman's correlation between therapist's SR and patient's IDAAP scores for final session (21 IUs)

\begin{tabular}{lccccc}
\hline & MIAffS & MIAffZ & IAffN/IAffP & IAffN/IAffZ & IAffN/IWRAD2 \\
\hline SR & $.518^{* *}$ & $.423^{*}$ & $-.502^{* *}$ & $.417^{*}$ & $.643^{* *}$ \\
\hline
\end{tabular}

Note. MIAffS = Mean of sum of affects. MIAffZ $=$ Mean of neutral affects. IAffN $=$ Mean negative affect. IAffP $=$ Mean of positive affects. IWRAD2 = Mean of referential activity. $S R=$ Speech rate.

${ }^{*} p<.05 ;{ }^{* *} p<.01$

negative affects at the same time as using words belonging to the reflection dictionary), whereas there is a significant negative correlation with the covariance between positive affects and referential activity scores (indicating that the therapist's speech is slower when, for example, the patient increases the production of positive terms of affect, thereby reducing those terms which would raise the scores of referential activity, and vice versa).

In the middle session many significant correlations can be seen. The first two, both negative, indicate that the therapist speaks more slowly both when the patient uses words that express emotional, physical or sensory experience (MISenS), and when he increases his referential activity (MIWRAD2). The third and fourth correlations present are in the positive, and each relates to the covariance between scales regarding affect. Both indicate that the therapist's SR is greater when the patient increases production of positive affect simultaneously with an increase either in the average of affect (IAffS) or with that of neutral affect (IAffZ). Finally, there is a negative correlation with the average of the covariation between affect and 'disfluency.' This shows that the therapist's speech slows down when the patient, in expressing more affect, loses fluency.

As we can see in Table 3, the first two correlations belonging to the final phase are both positive and relate to neutral affects and the total of affects; when both of these elements increase, so does the therapist's SR. Meanwhile the correlation linking SR and the covariation between positive and negative affect instead becomes negative, meaning the more the two diction- aries are used simultaneously, the more the therapist's speech slows down, and the less they are used simultaneously (or when the covariation becomes negative) the higher the therapist's SR. There is, however, another positive correlation with the covariations of negative affect (IAffN) and neutral affect (IAffZ) on the one hand, and with the covariation between negative affect and referential activity on the other.

Hypothesis 3: Relation between patient's SR and the therapist's RA. With the third working hypothesis we wanted to verify the presence of a correlation between the patient's SR and the scores of the four IDAAP dictionaries on the therapist's verbal production. The following table (Table 4) shows only the significant correlations for each of the three sessions.

At the sittings of the initial phase there is a negative correlation between the SR of the patient and the covariations between the neutral affects and the terms belonging to the reflection dictionary of the therapist, thereby indicating that the patient speaks more slowly when the therapist simultaneously increases the use of neutral emotional words and words referring to thought.

In the central session the patient's SR correlates positively with the therapist's use of words in the reflection dictionary. There are also two negative correlations with two covariations in the therapist's verbal production: firstly the covariation between positive affects and reflection, and secondly that between the average of affect and reflection (in both cases meaning that when the covariations increase in the therapist's verbal production, thus integrating these two dictionaries, the patient speaks more slowly).

Table 4. Values of Spearman's correlation between patient's SR and patient's IDAAP scores in the initial session (19 IUs), central session (49 IUs) and final session (21 IUs)

\begin{tabular}{|c|c|c|c|c|c|c|c|}
\hline & \multirow{2}{*}{$\begin{array}{c}\text { Initial session } \\
\text { IAffZ/IRef }\end{array}$} & \multicolumn{4}{|c|}{ Central session } & \multicolumn{2}{|c|}{ Final session } \\
\hline & & MIRef & IAffP/IRef & IAffS/IRef & IAffP/IWRAD2 & IAffN/IAffP & IAffS/IWRAD2 \\
\hline SR & $-.460^{*}$ & $.286^{*}$ & $-.477^{* *}$ & $.262^{*}$ & $.292^{*}$ & $.413^{*}$ & $-.414^{* *}$ \\
\hline
\end{tabular}

Note. IAffZ $=$ Mean of neutral affects. MIRef $=$ Mean of reflection words. IAffP $=$ Mean of positive affects. IAffS $=$ Mean of sum of affects. IWRAD2 $=$ Mean of referential activity. IAffN $=$ Mean of negative affects. $S R=S p e e c h$ rate. ${ }^{*} p<.05 ;{ }^{* *} p<.01$ 
Table 5. Values of Spearman's correlation between patient's SR and patient's SR for each session and for all sessions

\begin{tabular}{lcc}
\hline Session(s) & IUs $(n)$ & $r$ \\
\hline Initial session & 19 & $.620^{* *}$ \\
Central session & 49 & .040 \\
Final session & 21 & -.126 \\
All sessions & 89 & $.203^{*}$ \\
\hline${ }^{*} p<.05 ;^{* *} p<.01$ & &
\end{tabular}

Finally, there is a positive correlation of SR with the covariation between positive affects and referential activity. This increase indicates that when the therapist's verbal production shows a simultaneous increase in the use of these two dictionaries, the patient speaks faster.

In the session of the final phase, the patient's SR correlates negatively on the one hand with the covariation of negative and positive affect (when the therapist simultaneously increases the use of two dictionaries, the patient slows down his speech), while on the other hand it correlates with the covariation between the sum of affects and referential activity scores (meaning that if the therapist uses many terms of affect at the same time as increasing the amount of referential activity, the patient speaks more quickly).

Hypothesis 4: Relationship between the SR of the patient and that of the therapist. Finally, our goal was to verify the correlation between the two SR considering the three sessions either separately or together. The following table (Table 5) shows the resulting correlations both for the three sessions separately and for the three sessions together.

What you may notice is that in the session belonging to the initial phase the correlation between the two SRs is positive, spanning the IU that compose it.

In the central and final session there is no correlation, whilst taken in their entirety, there is a significant (positive) correlation between all the sessions.

\section{Discussion}

Relation between patient's SR and RA (Hypothesis 1). The data referring to the first working hypothesis confirms our expectations. Indeed, in all phases of therapy, the SR decreases when the patient increases the verbalization of subsymbolic aspects, both considered individually or in their covariation, while the SR increases when the patient makes more use of words belonging to the reflection dictionary either on their own or as part of a covariation between two scales. More specifically, the positive correlation between patient's SR and the IAffP/IRef covariation (mean of positive affects/mean of reflection words) may indicate the presence of a dissociated referential process which from a clinical point of view may give rise to defenses (e.g., rationalization). In turn, these defenses, through their inhibiting of referential connections, may require less processing time and thus be manifested by a higher rate of speech (as if the patient had switched to "automatic pilot," meaning that he is not actually listening to what he feels and trying to translate these feelings into words, but is simply doing what he has always done, that is avoiding contact with subsymbolic aspects which could potentially cause subjective pain).

Relation between the therapist's SR and the patient's RA (Hypothesis 2). The data seems to confirm the hypothesis, since in the early session and particularly in the central one the therapist's SR is lower when the patient verbalizes subsymbolic, affectiveemotional elements, or when the patient increases his disfluency. This latter result suggests that the therapist is sensitive to the patient's "difficulties in planning, lack of familiarity with a topic, and problems with lexical retrieval" (Campanelli, Bonfanti, \& Iberni, 2009, p. 35). Finally the therapist's SR increases when the patient verbalizes affect. Overall, therefore, we can say that this data is in line with expectations (Rocco, 2005, 2008), and that the correlations between the therapist's SR (a manifestation of his own referential process) and the patient's verbal production would seem to confirm that one variable affects the other (and that there could be a two-way interactive regulation) as an expression of synchronization between the two (according to Beebe \& Lachmann, 2002). If we look at this data in terms of a manifestation of the therapist's modulation towards the patient, then we can say that when the patient verbally expresses a positive covariation between the scales of affect (from which we can deduce that he is integrating his own referential process), the therapist increases the "pressure" by talking faster and, so to speak, by "riding the wave" with the patient, thereby actually legitimizing, in the intersubjective sense, the emotions which the patient is able to express (this, of course, would appear to work for this patient, but is not guaranteed to work for a patient with different characteristics). In other cases the therapist, in contrast, slows down his speech, and it can be assumed that by slowing his own referential process, he is trying to induce a similar process in the patient (given that, as we have seen, when the patient slows down his own speech he is able to produce significant subsymbolic, emotive/emotional material). In the third phase, at the end of therapy, some correlations change. We can assume that this is due to the fact that at this level, after the remission of symptoms and the achievement of therapeutic goals, the patient is already able to autonomously activate his own built-in referential process and therefore the therapist may to some extent be able to "tune-out."

Relationship between patient's SR and therapist RA (Hypothesis 3). The data tells us that the patient increases his referential process (lower SR) when the therapist uses words from the reflection dictionary, together (in a positive covariation) with those of affect. In our opinion this is of interest, especially since the correlation with a single scale of the reflec- 
tion dictionary is a positive one. In other words, there is a relation between the slowing of the patient's speech (which may indicate an increase in the quality of its referential process) and the simultaneous increase in terms used by the therapist relating to affective aspects and to thought (IREF, mean of reflection words), and not to thought alone.

At the same time there is an increase in the patient's SR when the therapist's verbal expression is a positive covariation between emotions and the average of referential activity. One way to interpret this data is the following: When the patient speaks quickly (moving away, as we have seen, from his subsymbolic and emotive nucleus), the therapist speaks more about affects, and at the same time increases referential activity. This could have the effect of gradually bringing the patient into contact with his/her subsymbolic aspects, until he/she starts to actually verbalize them.

The results however go in the same direction as the general hypothesis, confirming that SR could represent a paralinguistic parameter involved in the interactive regulation action.

Relation between patient's $S R$ and therapist's SR (Hypothesis 4). The variability of the obtained correlation can be seen as the expression by the therapist and patient in the clinical context of selfregulation (each maintains their own internal timing) and, during other moments, of interactive regulation (which finds its own expression, for example, in the session of the initial phase, during which a high correlation exists between the two), in the same way suggested by infant research studies (Jaffe, Beebe, Feldstein, Crown, \& Jasnow, 2001).

To sum up, all the results obtained seem to be in line with the study's basic hypotheses.

On the one hand we have seen that in the patient's verbal delivery there may be non-verbal indicators which convey information about his connection with subsymbolic, emotive/affective aspects, and on the other hand we have verified that significant links exist between the characteristics of the non-verbal production of one clinical subject and the characteristics of access to the (subsymbolic) emotive nuclei of the other (in the case of the patient, this access is closely related to the outcome of the psychotherapy). Moreover, the speed of the therapist's speech may be used from time to time to demonstrate emotional containment (if slow), relationship pressure (if increased), recognition of the affective state of others (if similar to that of the patient) and so on.

All these dynamics, which can be described in terms of mutual therapist/patient influence (interactive regulation), appear in the clinical setting to be expressed verbally, regardless of the content (which they themselves convey), and could play a key role in the result of the psychotherapeutic process.

From a non-verbal communication point of view, the data confirms that the therapist must be open and receptive, and as Bowbly (1991) suggests, "what cannot be communicated to the (m)other cannot be communicated to the self' (p. 295).

\section{Limitations of this study}

The present study is, of course, not without limitations. It only considers three sessions out of 14 , and to have more generalizable data it will be necessary to analyze a larger sample in order to ascertain their significance.

Moreover, the findings of this study need to be extended to a more in-depth level by applying the methodology to different types of psychotherapy (e.g., long-term psychotherapy) and with other types of patients (it would also be interesting to see what happens with a patient suffering from a borderline personality disorder, for example). This analytical methodology also needs to be applied to psychotherapy conducted by a different therapist, on the assumption that the ability to tune into the non-verbal channel of the patient, and use it from the therapeutic point of view, is personal, or dependant on the personality type, as well as on the intervention model adopted by the therapist.

Finally, the speech rate methodology currently presents two limitations. A first problem concerns the amount of time necessary to calculate SR; for example, for a fifty-minute session, more than 200 hours are needed (this means that more than 800 hours of work were needed to analyze the data of this study), not to mention the time needed for training. This necessarily limits the amount of data that can be collected.

Furthermore, it is important to note that in calculating speech rate, we recorded pauses in verbal production which exceeded 3 seconds as 3 -second pauses. This cutoff point had to be established, as discussed earlier, in order to prevent SR scores from being compromised by excessively long pauses due to reasons unrelated to cognitive or emotional processing. Although we believe this method was justified by the need to avoid confusing the referential process with, for instance, a deliberate resistance by the patient, we cannot be sure that for any given patient a longer than three-second pause is a reasonable time in which to process his/her own referential process. For the patient considered in this study this was not a particular issue, in fact pauses of more than three seconds only occurred a couple of times. Future research is needed in order to ascertain the methodological utility of this procedure using a larger sample.

\section{References}

Bauer, S., Tomicic, A., Martinez, C., Reinoso, A., \& Guzman, M. (2008, June). The voice as a psychotherapeutic instrument: The therapists's view. Poster session presented at the 39th International Annual Meeting of the Society for Psychotherapy Research (SPR), Barcelona, Spain.

Beebe, B., \& Lachmann, F. M. (2002). Infant Research and Adult Treatment: Co-Constructing Interactions. Hillsdale, NJ: The Analytic Press.

Benecke, C., Peham, D., \& Bänninger-Huber, E. (2005). Nonverbal relationship regulation in psychotherapy. Psychotherapy Research, 15(1-2), 81-90. doi: 10.1080/ 10503300512331327065

Boersma, P., \& Weenink, D. (2012). PRAAT: doing phonetics 
by computer [Computer program]. Version 5.3.14, retrieved 28 April 2012 from http://www.praat.org/

Bowbly, J. (1991). Post-script. In C. M. Parkes, J. StevensonHinde, \& P. Marris (Eds.), Attachment across the Life Cycle (pp. 293-297). London: Routledge.

Bucci, W. (1985). Dual coding: A cognitive model for psychoanalytic research. Journal of the American Psychoanalytic Association, 33(3), 571-607. doi: 10.1177/000306518503300305

Bucci, W. (1997a). Psychoanalysis and cognitive science. New York: The Guilford Press.

Bucci, W. (1997b). Patterns of discourse in 'good' and troubled hours. A multiple code interpretation. Journal of the American Psychoanalytic Association, 45(1), 155-187. doi: 10.1177/00030651970450010301

Bucci, W. (1999). The multiple code theory and the "third ear"; The role of theory and research in clinical practice. Psichiatria e Psicoterapia Analitica, 18(4), 299-310.

Bucci, W. (2011). The interplay of subsymbolic and symbolic processes in psychoanalytic treatment: It takes two to tango - but who knows the levels, who's the leader? The choreography of the psychoanalytic interchange. Psychoanalytic Dialogues, 21(1), 45-54. doi: 10.1080/10481885.2011.545326

Bucci, W., Kabasakalian-McKay, R., \& the RA Research Groups, Derner Institute Adelphi University, Garden City, N.Y. (2004, October). Scoring referential activity instructions for use with transcripts of spoken texts. Ethan A. Graham, First electronic edition.

Campanelli, L., Bonfanti, A., \& Iberni, E. (2009). Disfluenza: cos'è e che farsene. Introduzione al pacchetto I-DAAP per la sua misurazione [Disfluency: what it is and how to use it]. Quaderni CPD, 6, 35.

Davanloo, H. (1990). Unlocking the Unconscious. Chichester, England: John Wiley \& Sons Ltd.

De Coro, A., Mariani, R, Pazzagli, C., Andreassi, S., Ortu, F., \& Caviglia, G. (2004, October). Il nuovo dizionario computerizzato per la valutazione dell'attività referenziale. Un'applicazione allo studio del caso singolo [The new computerized dictionary for the evaluation of referential activity. An application to a single-case study]. Paper presented at the 6th National Congress AIP, Aosta.

De Coro, A., Ortu, F., Caviglia, G., Andreassi, S., Pazzagli, C., Mariani, R., ..., \& Maskit, B. (2004, Giugno). Referential activity and verbal exchange in psychotherapy: A first clinical application of the italian WRAD version to the study of a single case. Paper presented at 35th annual meeting of the Society for Psychotherapy Research, Roma.

Derogatis, L. R. (1983). Administration, scoring, and procedures manual for the recise version. Baltimore: Clinical Psychometric Research.

Flegenheimer, W. (1982). Techniques of Brief Psychotherapy. New York: Jason Aronson.

Fogliato, E., Strappa, V., Branchini, S., \& Rapisarda, F. (2009). L'attività referenziale [Referential activity]. In M. Vigorelli (Ed.), Laboratorio didattico per la ricerca in psicoterapia [Didactic laboratory for psychotherapy research] (pp. 275-310). Milano:Raffaello Cortina.

Fosha, D. (2000). The transforming power of affect. New York: Basic Books.

Horiwitz, L. M., Sampson, H., Siegelman, E. Y., Wolfson, A., \& Weiss, J. (1975). On the identification of warded-off mental contents: An empirical and methodological contribution. Journal of Abnormal Psychology, 8(5), 545-558. doi: 10.1037/h0077139

Jaffe, J., Beebe, B., Feldstein, S., Crown, C., \& Jasnow, M. (2001). Rhythms of dialogue in early infancy. Monographs of the Society for Research in Child Development, 66(2), 1-132. doi: 10.1111/1540-5834.00143

Karasu, T. B. (1986). The specificity versus non-specificity dilemma: Toward identifying therapeutic change agents.
American Journal of Psychiatry, 143(6), 687-695.

Mariani, R., \& De Coro, A. (2013). Study of a short-term treatment by referential activity linguistic measures. Research in Psychotherapy: Psychopathology, Process and Outcome, 16(1), 42-53.

Mergenthaler, E., Freni, S., Giampieri, E., \& Ferrari, R. (1998). Regole standardizzate di trascrizione delle sedute di psicoterapia [Standardized rules of transcription of psychotherapy sessions]. Ricerca in Psicoterapia, 1(1), 29--46.

Merten, J. (2005). Facial microbehavior and the emotional quality of the therapeutic relationship. Psychotherapy Research, 15(3), 325-333. doi: 10.1080/10503300500091272

Mitchell, S. (2000). Relationality: From attachment to intersubjectivity. Hillsdale, NJ: The Analytic Press Inc.

Moneta, M. E., Penna, M., Loyola, H., Buchheim, A., \& Kächele, H. (2008). Measuring emotion in the voice during psychotherapy interventions: A pilot study. Biological Research, 41(4), 389-395. doi: 10.4067/S0716-97602008000400004

Raue, P. J., Goldfried, M. R., \& Barkham, M. (1997). The therapeutic alliance in psychodynamic-interpersonal and cognitive-behavioral therapy. Journal of Consulting and Clinical Psychology, 65(4), 582-587. doi: 10.1037/0022-006X.65.4.582

Rice, L., \& Wagstaff, A. (1967). Client voice quality and expressive style as indexes of productive psychotherapy. Journal of Consulting Psychology, 31(6), 557-563. doi: 10.1037/h0025164

Rice, L., \& Kerr, G. P. (1986). Measures of client and therapist vocal quality. En L.S. Greenberg \& W. M. Pinsof (Eds.), The Psychotherapeutic Process: A Research Handbook (pp. 73105). New York: Guilford.

Rocco, D. (2005). Analisi degli aspetti paraverbali in una psicoterapia dinamica breve: Il metodo dell'Attività Referenziale e l'analisi della Speech Rate [Analysis of non-verbal aspects in a short-term dynamic psychotherapy: The method of Referential Activity and the analysis of Speech Rate]. Ricerca in Psicoterapia, 8(1), 127-147.

Rocco, D. (2008). Analisi empirica dell'influenzamento reciproco tra paziente e terapeuta. Il linguaggio dei contenuti ed il linguaggio delle emozioni [Empirical analysis of mutual influencing between therapist and patient. The language of contents ad the language of emotions]. Ricerca in Psicoterapia, 11(1), 47-74.

Stern, D. N. (2004), The Present Moment in Psychotherapy and Everyday Life. New York: Norton \& Company, Inc.

Stolorow, R. D., \& Atwood, G. E. (1992). Contexts of Being: The Intersubjective Foundations of Psychological Life. New York: The Analytic Press.

Tomicic, A., Martinez, C., Altimir, C., Bauer, S., \& Reinoso, A. (2009) Coordinación vocal como una dimensión de la regulación mutua en psicoterapia. Revista Argentina de Clinica Psicológica, 18(1), 31-41.

Tronick, E. Z., Bruschweiler-Stern, N., Harrison, A. M., LyonsRuth, K., Morgan, A. C., Nahum, J. P., Sander, L., \& Stern, D. N. (1998), Dyadically expanded states of consciousness and the process of therapeutic change. Infant Mental Health Journal, 19(3), 290-299. doi: 10.1002/(SICI)1097-0355(199823) 19:3<290::AID-IMHJ4>3.0.CO;2-Q

Westen, D., Shelder, J., \& Lingiardi, V. (2003) La valutazione della personalità con la SWAP-200 . [Assessing Personality with SWAP-200]. Milano: Raffaello Cortina.

Wiseman, H., \& Rice, L. (1989). Sequential analyses of therapist-client interaction during change events: a task-focused approach. Journal of Consulting and Clinical Psychology, 57(2), 281-286. doi: 10.1037/0022-006X.57.2.281

Received June 20, 2012

Revision received July 7, 2012 Accepted September 24, 2012 